\title{
Syndecan-1 in Breast Cancer Stroma Fibroblasts Regulates Extracellular Matrix Fiber Organization and Carcinoma Cell Motility
}

\author{
Ning Yang, ${ }^{*}$ Rachel Mosher, ${ }^{\dagger}$ Songwon Seo, ${ }^{\ddagger}$ \\ David Beebe, ${ }^{\dagger}$ and Andreas Friedl ${ }^{\star} \S$ \\ From the Departments of Pathology and Laboratory Medicine,* \\ and Biomedical Engineering, College of Engineering, ${ }^{\dagger}$ and the \\ Department of Biostatistics and Medical Informatics, ${ }^{\ddagger}$ University \\ of Wisconsin-Madison, Madison, and the Laboratory Medicine \\ Service, William S. Middleton Memorial Veterans Hospital, \\ Madison, Wisconsin
}

Stromal fibroblasts of breast carcinomas frequently express the cell surface proteoglycan syndecan-1 (Sdc1). In human breast carcinoma samples, stromal Sdc1 expression correlates with an organized, parallel, extracellular matrix (ECM) fiber architecture. To examine a possible link between stromal Sdc1 and the fiber architecture, we generated bioactive cell-free three-dimensional ECMs from cultures of Sdc1-positive and Sdc1-negative murine and human mammary fibroblasts (termed ECM-Sdc1 and ECM-mock, respectively). Indeed, ECM-Sdc1 showed a parallel fiber architecture that contrasted markedly with the random fiber arrangement of ECM-mock. When breast carcinoma cells were seeded into the fibroblast-free ECMs, ECM-Sdc1, but not ECM-mock, promoted their attachment, invasion, and directional movement. We further evaluated the contribution of the structural/compositional modifications in ECM-Sdc1 on carcinoma cell behavior. By microcontact printing of culture surfaces, we forced the Sdc1-negative fibroblasts to produce ECM with parallel fiber organization, mimicking the architecture observed in ECM-Sdc1. We found that the fiber topography governs carcinoma cell migration directionality. Conversely, an elevated fibronectin level in ECM-Sdc1 was responsible for the enhanced attachment of the breast carcinoma cells. These observations suggest that Sdc1 expression in breast carcinoma stromal fibroblasts promotes the assembly of an architecturally abnormal ECM that is permissive to breast carcinoma directional migration and invasion. (Am J Pathol 2011, 178:325-335; DOI: 10.1016/j.ajpath.2010.11.039)

Epithelial-stromal interactions play crucial roles in directing mammary gland development and in maintaining normal tissue homeostasis. Conversely, during tumorigenesis, the stroma accelerates carcinoma growth and progression. The predominant cell type within the stromal compartment is the fibroblast, which synthesizes, organizes, and maintains a three-dimensional (3D) network of glycoproteins and proteoglycans known as the extracellular matrix (ECM). Normal stromal fibroblasts and their ECM are believed to exert an inhibitory constraint on tumor growth and progression. ${ }^{1,2}$ Major alterations occur in the stromal fibroblasts and ECM during neoplastic transformation, giving rise to a permissive and supportive microenvironment for carcinomas. Compared with their quiescent normal counterpart, carcinoma-associated fibroblasts display an activated phenotype, which is characterized by the expression of smooth muscle markers, an enhanced proliferative and migratory potential, and altered gene expression profiles. Carcinoma-associated fibroblasts produce and deposit elevated amounts and abnormal varieties of ECM components. ${ }^{3-5}$ Recent evidence $^{6,7}$ indicates that not only ECM composition but also ECM architecture are altered in carcinomas and that these changes may promote tumor progression. However, the contribution of these stromal modifications to tumor development and the molecular mechanisms and signaling events underlying these alterations are incompletely understood.

Supported by the National Institutes of Health (grant R01 CA107012).

Accepted for publication July 29, 2010.

The contents of this article do not represent the views of the Department of Veterans Affairs or the US government.

CME Disclosure: The authors did not disclose any relevant financial relationships.

Address reprint requests to Andreas Friedl, M.D., Department of Pathology and Laboratory Medicine, University of Wisconsin-Madison, 6051 Wisconsin Institutes for Medical Research (WIMR), 1111 Highland Ave, Madison, WI 53705-2275. E-mail: afriedl@wisc.edu. 
Syndecans (Sdcs) constitute a family of transmembrane heparan sulfate proteoglycans with four known members (Sdc1-4). Via their heparan sulfate glycosaminoglycan (HS-GAG) chains, Sdcs interact with a wide variety of proteins, including growth factors and ECM constituents. ${ }^{8-10}$ Consequently, they play roles in cell growth, adhesion, migration, and morphogenesis. Sdc2 appears to be required to assemble laminin and fibronectin (FN) into a fibrillar matrix. ${ }^{11}$ Syndecan-4 has also been implied to participate in FN matrix assembly. Concomitant engagement of Sdc4 and integrins promotes Rho GTPase and focal adhesion kinase (FAK) activities, which are crucial for efficient initiation of FN matrix assembly. ${ }^{12-15}$ Sdc1 is expressed primarily by epithelial and plasma cells of healthy adult tissue. ${ }^{16}$ Recently, we and others ${ }^{17,18}$ observed the induction of Sdc1 in stromal fibroblasts of invasive breast carcinomas. Syndecan-1, aberrantly expressed by stromal fibroblasts in breast carcinomas, participates in a reciprocal carcinoma growthpromoting feedback loop that requires proteolytic shedding of its ectodomain. ${ }^{17,19,20}$ Although the role of Sdc1 in matrix assembly has not been investigated, this molecule has interacted with various ECM components, including FN, fibrillar collagens, laminin, vitronectin, thrombospondin, and tenascin. ${ }^{8-10}$

In the present study, we explore the possibility that Sdc1 expression by stromal fibroblasts may be causally involved in altered matrix production of tumor stroma. We find that in mammary stromal fibroblasts, Sdc1 regulates ECM assembly and determines ECM fiber architecture. We further show that cell-free 3D ECMs produced by Sdc1-expressing fibroblasts facilitate the directional migration of mammary carcinoma cells and link this activity to the parallel fiber architecture.

\section{Materials and Methods}

\section{Human Breast Carcinoma Samples}

Paraffin sections from a tissue microarray containing duplicate tumor samples from 207 patients with breast carcinoma were immunoperoxidase labeled with an antibody to Sdc1, as previously described. ${ }^{17}$ The immunolabeled slides were examined by bright field microscopy and scored manually, using a method developed by Harvey and coworkers. ${ }^{21}$ Sections from the same tumor blocks and those from MMTV-Wnt1-induced mammary tumors (provided by C. Alexander, Ph.D.), were stained with picro-sirius red (sirius red F3B [C.I. 35782; $0.1 \% \mathrm{w} / \mathrm{v}]$ in saturated picric acid aqueous solution) for 60 minutes, followed by two washes in glacial acetic acid $(0.5 \% \mathrm{v} / \mathrm{v})$. Images acquired during polarization microscopy were overlaid with a predesigned template that defined nine evenly distributed measurement points. The fiber closest to each of these points was identified, and the intersection angle of the nearest crossing fiber was measured using an angle measurement tool (ImageJ; http://rsbweb.nih.gov/ij/). Two observers measured the angles in the tissue microarray slides independently in a blinded fashion (intraclass correlation coefficient, 0.82).

\section{Cell Culture}

The human breast carcinoma cell lines MDA-MB-231 were from A.C. Rapraeger, T47D cells were from M. Gould, Ph.D., and NIH-3T3 fibroblasts were from J.S. Malter, MD (University of Wisconsin-Madison). Immortalized human mammary fibroblasts (HMFs) (originally called RMF-EG and HMF herein) were generously provided by $C$. Kuperwasser, Ph.D. ${ }^{2}$ Cells were maintained in Dulbecco's modified Eagle's medium supplemented with either $10 \%$ fetal bovine serum (T47D cells) or 10\% fetal calf serum (all other cells), 2-mmol/L L-glutamine, and 100-U/ml penicillin and streptomycin.

\section{Stable Transfections}

The NIH-3T3 cells stably transfected with mouse Sdc 1 were previously described. ${ }^{17}$ The HMF cells were stably transfected with a pcDNA3.1 vector containing the cDNA of mouse Sdc1 (a gift from A.C. Rapraeger, Ph.D) or empty vector using a commercially available system (Amaxa Nucleofection System; Lonza, Walkersville, MD) according to the manufacturer's instructions. After transfection, cells were selected with $500-\mu \mathrm{g} / \mathrm{ml}$ G418 and cells expressing Sdc1 at high levels were enriched by fluorescence-activated cell sorting. The HMF mock and Sdc1 cells were maintained in medium containing $250-\mu \mathrm{g} / \mathrm{ml} \mathrm{G} 418$.

\section{Matrix Production}

Fibroblast-derived 3D ECM was prepared according to the protocol developed by Cukierman. ${ }^{22,23}$ Briefly, NIH3 T3 and HMF cells were cultured in a highly confluent state for 7 days in the presence of $50-\mu \mathrm{g} / \mathrm{ml}$ ascorbic acid (Fisher Scientific Inc., Pittsburgh, PA). The matrix cultures were then treated with alkaline detergent solution (25-mmol/L Tris- $\mathrm{HCl}, \mathrm{pH} 7.4 ; 150-\mathrm{mmol} / \mathrm{L}$ sodium chloride; $0.5 \%$ Triton $\mathrm{X}-100$; and $20-\mathrm{mmol} / \mathrm{L}$ ammonia hydroxide) to remove the fibroblasts. Cellular remnants were washed away with PBS, leaving an intact 3D cell-free ECM attached to the culture surface.

\section{Immunofluorescence Staining}

Unextracted 3D cultures were fixed/permeabilized in 4\% paraformaldehyde and $0.5 \%$ Triton X-100 in PBS for 5 minutes at room temperature and then fixed with $4 \%$ paraformaldehyde for an additional 15 minutes. Cells were blocked with $5 \%$ fetal bovine serum in PBS for 1 hour at room temperature and then incubated with mouse anti-FN (1 $\mu \mathrm{g} / \mathrm{ml}$; BD Biosciences, San Jose, CA) and rat anti-mouse Sdc1 antibody $(5 \mu \mathrm{g} / \mathrm{ml}$; a gift from A.C. Rapraeger, Ph.D) at $4^{\circ} \mathrm{C}$ overnight. The cell-free 3D ECMs were directly blocked with $5 \%$ fetal bovine serum and incubated with primary antibodies overnight. After washing with PBS, goat anti-mouse IgG (Alexa Fluor488-conjugated) and goat anti-rat IgG (Alexa Fluor647-conjugated) (both $5 \mu \mathrm{g} / \mathrm{ml}$; Invitrogen, Carlsbad, CA) were added to 3D culture or cell-free ECM for 1 hour at room temperature. The preparations were analyzed with a laser-scanning confocal microscope (MRC 1024; Bio-Rad Laboratories, Inc., Hercules, CA). 


\section{Dot and Western Blots}

Whole-cell lysates of NIH-3T3 and HMF cells were prepared using a radioimmunoprecipitation assay (RIPA) buffer (Boston BioProducts, Worcester, MA). The ECM proteins of cell-free ECM-mock and ECM-Sdc1 were solubilized using TUT buffer (8-mol/L urea, 10-mmol/L Tris, 1-mmol/L sodium sulfate, and $0.1 \%$ Triton X-100, $\mathrm{pH} 8.0$ ). Equal amounts of protein lysates were either blotted directly onto the nitrocellulose membranes or fractionated on $4 \%$ to $12 \%$ precast gel (Criterion XT; Bio-Rad Laboratories, Inc.) before transfer to polyvinylidene difluoride membranes. The membranes were probed overnight with rat anti-Sdc1 $(1-\mu \mathrm{g} / \mathrm{ml})$ or mouse anti-FN $(0.25-\mu \mathrm{g} / \mathrm{ml})$ antibodies. Horseradish peroxidase-conjugated anti-rat (20-ng/ml) or anti-mouse (100-ng/ml) lgG (Sigma, St. Louis, MO) was used as a secondary antibody. FN or Sdc1 was then visualized using a maximum sensitivity substrate SuperSignal West Femto (Pierce, Thermo Fisher Scientific, Rockford, IL).

\section{ECM Fiber Angle Measurements}

Confocal images of the cell-free ECMs were overlaid with a predesigned template that defined nine evenly distributed measurement points. Fiber-to-fiber angles were measured as previously described for the human tumor samples. A minimum of 10 images were analyzed for each condition.

\section{Cell Growth Assay}

The MDA-MB-231 $\left(2 \times 10^{4}\right.$ cells $)$ and T47D $\left(5 \times 10^{4}\right.$ cells) were plated on cell-free $\mathrm{NIH}-3 \mathrm{~T} 3$ and HMF mock and Sdc1 ECMs and cultured for up to 6 days. Carcinoma cells were collected every day by trypsinization and counted with a hemocytometer.

\section{Cell Attachment Assay}

Semiconfluent cultures of MDA-MB-231 and T47D cells were stained with a nuclear dye (Hoechst 33342; 2.5 $\mu \mathrm{g} / \mathrm{ml}$; Invitrogen, Carlsbad, CA). Labeled cells were added to the glass-bottom dishes (MatTek Corporation, Ashland, MA) precoated with $\mathrm{NIH}-3 \mathrm{~T} 3$ or HMF-derived ECM-mock, ECM-Sdc1, or soluble FN and incubated at $37^{\circ} \mathrm{C}$ for 10 minutes. The unattached cells were removed by washing with PBS, and attached cells were fixed with ice-cold $100 \%$ methanol. Images of the nuclei were acquired using an inverted microscope. The number of attached cells was determined using ImageJ software (ImageJ), and the average value obtained on the FN control was normalized as 1 arbitrary unit.

\section{Invasion Assay}

The NIH-3T3 or HMF cells were cultured in the insert of invasion chambers (BD Biocoat Matrigel Invasion Chambers; BD Biosciences) for 7 days and then extracted to leave cell-free ECMs on the upper side of the insert. MDA-MB-231 cells were loaded onto these fibroblast- derived ECMs and cultured at $37^{\circ} \mathrm{C}$ for 24 hours in Dulbecco's modified Eagle's medium supplemented with $2 \%$ fetal calf serum. The lower chambers were filled with DMEM (Dulbecco's modified Eagle's medium) containing $10 \%$ fetal calf serum as a source of chemoattractants. Noninvading MDA-MB-231 cells remaining on the upper side of the insert were removed, whereas the invading cells attached to the lower side of the insert were fixed with $100 \%$ methanol and then stained with Hoechst dye 33342. Images of the nuclei were acquired using an inverted microscope (Olympus) and analyzed using ImageJ software.

\section{Cell Migration Analysis (Time-Lapse Motility} Assay)

Live MDA-MB-231 cells were labeled using a cytoplasmic membrane staining kit (CellBrite; Biotium Inc., Hayward, CA) according to the manufacturer's instructions. Labeled cells were added to glass-bottom tissue culture plates precoated with $\mathrm{NIH}-3 \mathrm{~T} 3$ and HMF ECMs. After overnight incubation, the plates were placed in the environmentally controlled chamber of a confocal bioimager (BD Pathway; BD Biosciences). Cell movements were monitored in real time for 5 to 6 hours, and images were captured every 30 minutes with a cooled 12-bit chargecoupled device camera (Autovision; BD Biosciences). The resulting images were stacked using ImageJ software (ImageJ). The migratory directionality of each individual cell was determined by tracing the path of the manually detected cell center using the Fragment Line tool in ImageJ. The results represent the average of two to three independent experiments analyzing 50 to 100 cells each.

\section{Microcontact Printing}

Elastomeric polydimethylsiloxane stamps with line or square patterns were fabricated in the Beebe laboratory. After optimization, lines with a width of $150 \mu \mathrm{m}$ and spacing of $150 \mu \mathrm{m}$ were used. The size of squares used as controls was $450 \times 450 \mu \mathrm{m}$, and the spacing between adjacent squares was $150 \mu \mathrm{m}$. These stamps were used to print FN patterns directly onto the glass-bottom tissue culture dishes. ${ }^{24}$ Briefly, the stamps were cleaned by sonication, immersed in FN solution (50 $\mu \mathrm{g} / \mathrm{ml}$ in PBS) for 2 hours to allow for protein adsorption, and then air dried in a tissue culture hood. The FN-coated stamps were placed onto the glass bottom of the culture dishes, pressed down gently, and kept in this position for approximately 15 minutes to ensure the transfer of FN onto the culture dishes. After the removal of the stamps, the dishes were blocked with $5 \%$ heat-inactivated fetal bovine serum for 1 hour. The HMF mock and Sdc1 cells were then added to the patterned dishes and incubated for 20 minutes at $37^{\circ} \mathrm{C}$. The HMF cells preferentially attached to the FN patterns, leaving the spacing regions unoccupied. After washing away the unattached cells, the remaining cells were cultured for 7 days to produce 3D ECMs (see previous data). 


\section{Statistics}

Either the Student's $t$-test or one-way analysis of variance was used, depending on the number of groups to be compared. When analysis of variance yielded significance compared with multiple groups, pairwise comparisons using the Tukey's test were performed. Fiber angle data from the mouse tissues and from ECMs produced in vitro were compared with the nonparametric Mann-Whitney $U$ test. Spearman correlation analysis was used to examine the relationship between angle measurements in human tumor tissue microarray and tumor parameters on a continuous scale. Statistical significance was defined as a two-tailed $P<0.05$.

\section{Results}

\section{Stromal Sdc1 Expression Correlation with Parallel Stromal Fiber Architecture in Human and Mouse Mammary Carcinomas}

We and others ${ }^{17,18}$ have previously reported that Sdc1 is aberrantly expressed by stromal fibroblasts in human breast carcinomas. During microscopic examination of breast carcinoma tissues, it appears that Sdc1 expres-
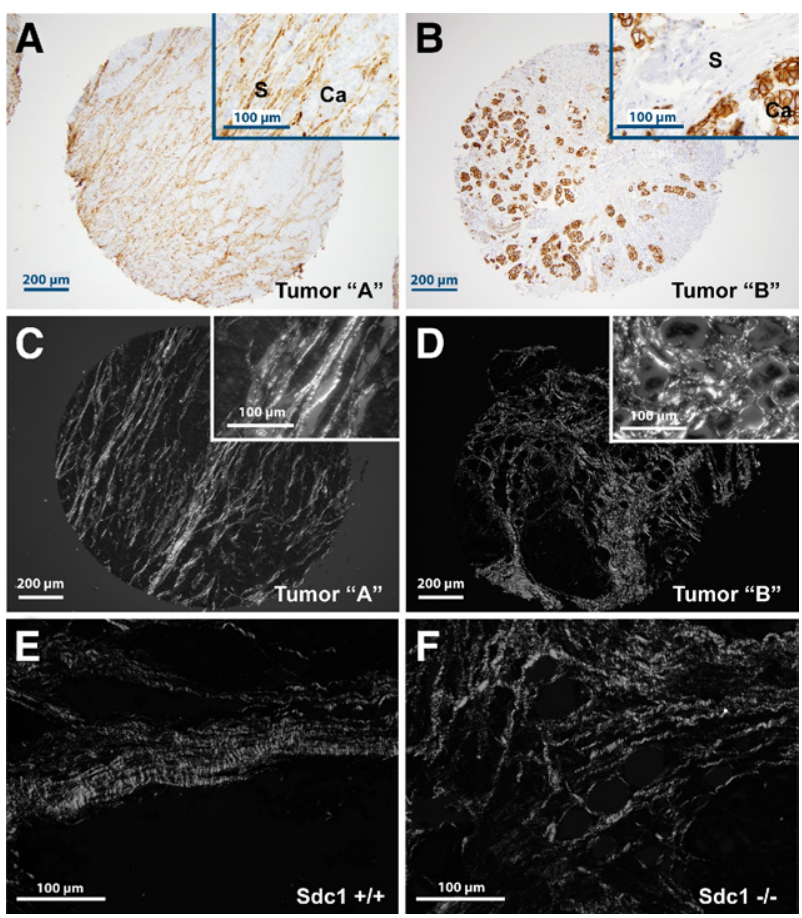

Figure 1. In human breast carcinoma samples, stromal Sdc1 expression correlates with a parallel ECM fiber architecture. A and B: Paraffin sections of human breast carcinoma samples were immunolabeled with an antibody to $\mathrm{Sdc1}$. In tumor A, the stroma (S) is strongly Sdc1 positive, whereas the carcinoma cells ( $\mathrm{Ca}$ ) are $\mathrm{Sdc} 1$ negative. In tumor $\mathrm{B}$, carcinoma cells are Sdc1 positive but the stroma is Sdc1 negative. Insets: Representative areas at higher magnification. $\mathbf{C}$ and $\mathbf{D}$ : The tissue microarray sections were stained with the picro-sirius red method and examined by polarization microscopy. The sirius red dye binds primarily to collagen. The same tumor cores as in $\mathbf{A}$ and $\mathbf{B}$ are shown. Insets: Representative areas at higher magnification. $\mathbf{E}$ and F: Paraffin sections from mammary tumors arising in MMTV-Wnt1-transgenic mice with an $\mathrm{Sdc}^{+/+}$or an $\mathrm{Sdc}^{-/-}$background were stained with the picro-sirius red method, as described for $\mathbf{C}$ and $\mathbf{D}$.
Table 1. ECM Fiber-to-Fiber Angles in Human Breast Carcinomas*

\begin{tabular}{ccc}
\hline Stroma & Human & Mouse \\
\hline Sdc1 $_{\text {positive }}{ }^{+}$ & $43.8 \pm 10.3$ & $24.4 \pm 7.2$ \\
Sdc1 negative $^{\dagger}$ & $47.2 \pm 9.9$ & $35.0 \pm 12.8$ \\
$P$ value & 0.024 & 0.011 \\
\hline
\end{tabular}

*These angles were dichotomized along the median into stroma Sdc 1 positive $(\mathrm{H}$-score $\geq 5)$ and Sdc1 negative $(\mathrm{H}$-score $<5)$ and in MMTVWnt1 mouse mammary tumors arising in an Sdc1 $1^{+/+}$and an Sdc1 $1^{-1}$ genetic background.

tData are given as mean $\pm \mathrm{SD}$

sion in fibroblasts is frequently associated with an organized desmoplastic stroma. To confirm this subjective impression, we examined a previously characterized tissue microarray that contains duplicate tumor tissue samples from 207 patients with breast carcinoma. ${ }^{25,26}$ Stromal Sdc1 was detected by immunohistochemistry and quantified by manual scoring ${ }^{21,25}$ (Figure 1, A and B). The ECM fiber architecture was visualized by polarization microscopy of picro-sirius red-stained paraffin sections $^{27}$ (Figure 1, C and D). Because of tissue loss or absence of identifiable ECM fibers, 21 tumors had to be eliminated from the analysis. The ECM fiber-to-fiber angles (mean \pm SD; $45.6 \pm 10.1$ degrees; $n=186$ ) correlated negatively with stromal Sdc1 expression $(r=$ $-0.21 ; P=0.004)$. As expected, when the tumors were dichotomized along the median into Sdc1- positive and Sdc1-negative cases, the fiber angles were more acute in carcinomas with Sdc1-positive stroma (Table 1). Conversely, the ECM fiber-to-fiber angles did not correlate with any other tumor parameter, including size, grade, lymph node status, Ki-67 proliferative index, estrogen or progesterone receptor expression, human epidermal growth factor receptor 2 (Her-2) overexpression, or patient age. To examine more rigorously a potential link between stromal Sdc1 and ECM organization, we compared the ECM fiber architecture in MMTV-Wnt1-induced mouse mammary tumors arising in genetically Sdc1-deficient and wild-type animals. In this animal model, we have previously demonstrated that strong Sdc1 induction in stromal fibroblasts occurs in the abundant stroma. ${ }^{17}$ Similar to the human breast carcinomas, the fiber-to-fiber angles were significantly $(P<0.011)$ more acute in tumors arising in Sdc1 $1^{+/+}$animals (Figure 1E and Table 1) than in tumors from Sdc1-l- mice (Figure 1F). These findings in human and mouse tumors suggest a modulatory effect of Sdc1 on tumor stroma ECM organization.

\section{Fibroblast-Derived 3D ECMs in Vitro: Sdc1 Regulates Fiber Architecture}

To model ECM production in vitro, we used an experimental system developed by Amatangelo and co-workers. ${ }^{22,28,29}$ In this model, fibroblasts are permitted to grow to an overconfluent state under culture conditions that favor ECM production. The fibroblasts are then removed by detergent extraction, leaving a cell-free 3D ECM scaffold behind, which can then be reseeded with other cell types. We studied ECMs produced by generic mouse 
A

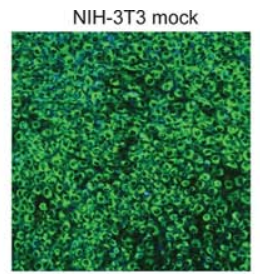

B
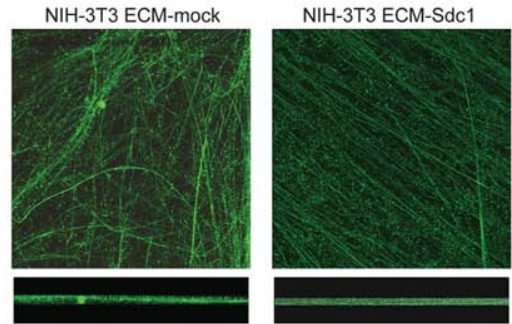

C

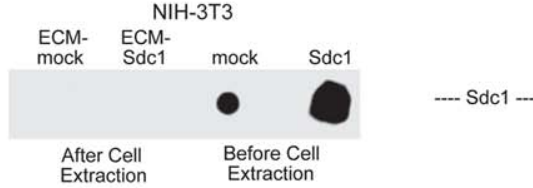

D

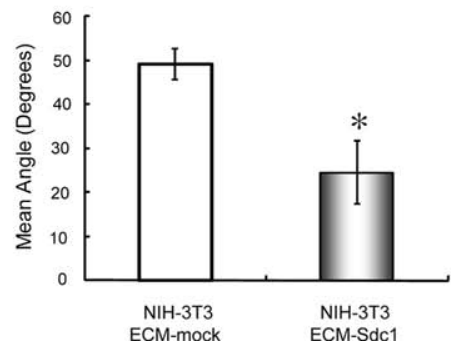

NIH-3T3 Sdc1

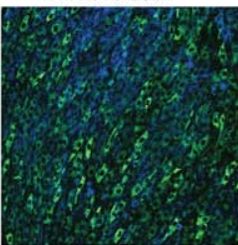

NIH-3T3 ECM-Sdc1
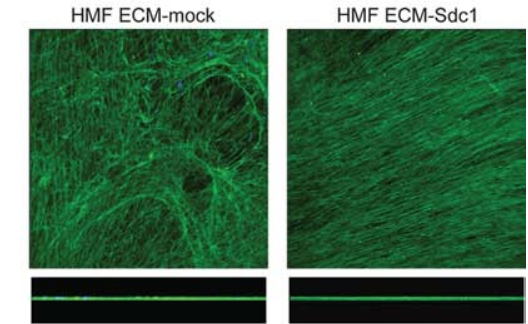

HMF
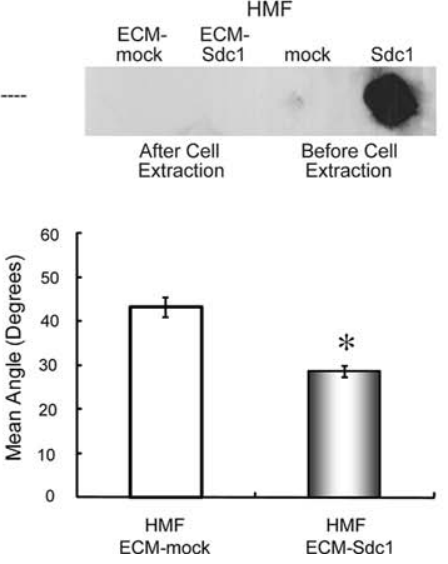

Figure 2. The ECMs produced by Sdc1-expressing fibroblasts are organized in a parallel fibe pattern. A and B: Generation of Sdc1-expressing fibroblasts and fibroblast-derived 3D ECMs. Both FN (green signal) and Sdc1 (blue signal) were antibody labeled and detected by fluorescence microscopy (original magnification, $\times 200$ ). A Representative confocal images of long-term 3D cultures of NIH-3T3 mock, NIH-3T3 Sdc1, HMF mock, and HMF Sdc1 fibroblasts. B: The ECMs after cell extraction. Bottom: Z-axis views $\left(90^{\circ}\right.$ side views) of the cell-free ECMs. C: Dot blot analysis of Sdc1 protein in unextracted 3D cultures of NIH-3T3 mock, NIH-3T3 Sdc1, HMF mock, and HMF Sdc1 fibroblasts and in the cellfree ECMs derived from these cells. D: The ECM fiber angles were measured as described in $\mathrm{Ma}$ terials and Methods. The mean fiber-to-fiber angles of NIH-3T3 and HMF-derived ECM-mock and ECM-Sdc1 are depicted. Error bars indicate SDs; statistically significant differences at ${ }^{*} P<$ 0.0001
NIH-3T3 cells and organotypic immortalized HMFs. ${ }^{2}$ The induction of Sdc1 in fibroblasts of breast carcinoma stroma was simulated in vitro by stable forced expression of Sdc1. The expression of Sdc1 in transfected cells was confirmed by immunofluorescence staining (Figure 2A) and dot blot analysis (Figure 2C). After detergent extraction of fibroblasts, cell-free ECMs remained attached to the culture vessel.

To characterize the ECMs, they were immunofluorescently labeled with antibodies to $\mathrm{FN}$ and examined by laser confocal microscopy (Figure 2B). FN immunolabeling proved a robust method to visualize ECM fibers, although labeling with antibodies to collagen I yielded similar results (data not shown). The thickness of the ECMs produced by Sdc1-positive and Sdc1-negative fibroblasts (subsequently referred to as ECM-Sdc1 and ECM-mock, respectively, herein) was uniform throughout the culture vessel and averaged $34 \mu \mathrm{m}$ for $\mathrm{NIH}$-3T3 ECMs and $14 \mu \mathrm{m}$ for HMF (z-axis views of the cell-free matrices, Figure 2B). Importantly, Sdc1 was undetectable in the ECMs by immunofluorescence microscopy (Figure 2B) and dot blot analysis (Figure 2C), demonstrating that the cell extraction had effectively removed Sdc1 with the fibroblast cell membranes.

The fiber architecture appeared dramatically different between ECM-mock and ECM-Sdc1. The ECM-Sdc1 from both $\mathrm{NIH}-3 \mathrm{~T} 3$ and HMF cells displayed an orga- nized parallel FN fiber orientation (Figure 2B) similar to the fiber architecture found in Sdc1-positive stroma in vivo (Figure 1, A and C) and contrasting with the haphazard fiber arrangement in the corresponding ECM-mock (Figure 2B). Quantitative analysis revealed that the mean fiber-to-fiber angles were significantly smaller in ECM-
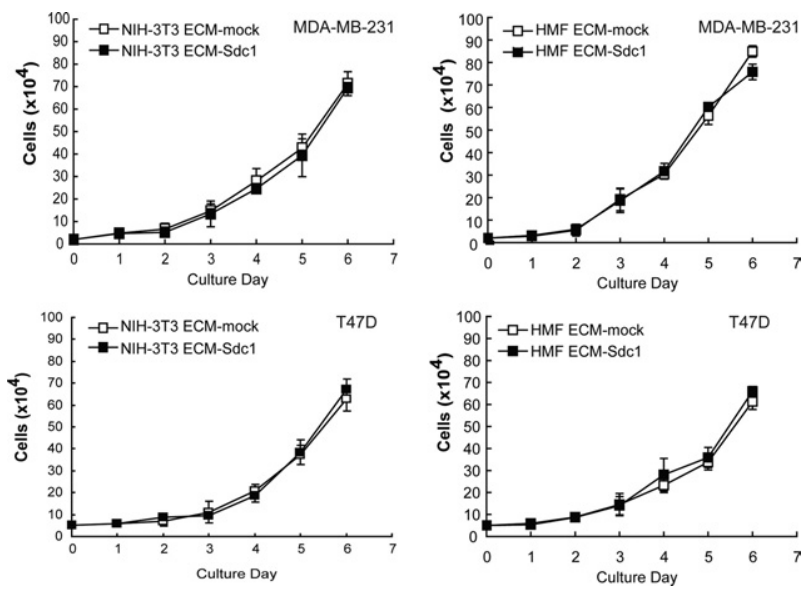

Figure 3. The ECMs produced by Sdc1-expressing fibroblasts do not stimulate breast carcinoma cell proliferation. Growth of MDA-MB-231 cells and T47D cells in NIH-3T3 and HMF ECMs was monitored during a 6-day culture period. Each point of the curve represents the average of two to three independent experiments. Error bars indicate the SDs. 
A
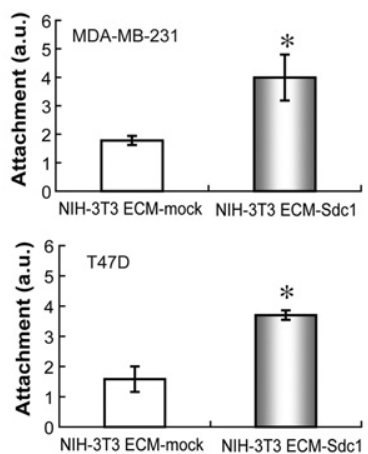

B

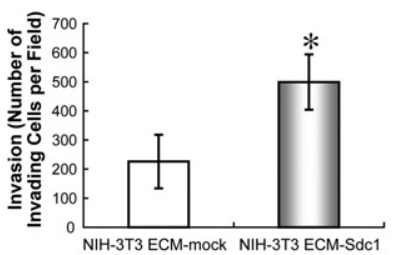

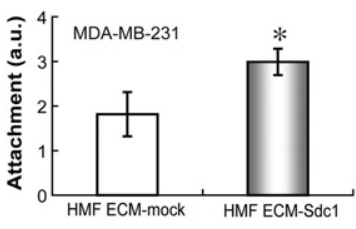
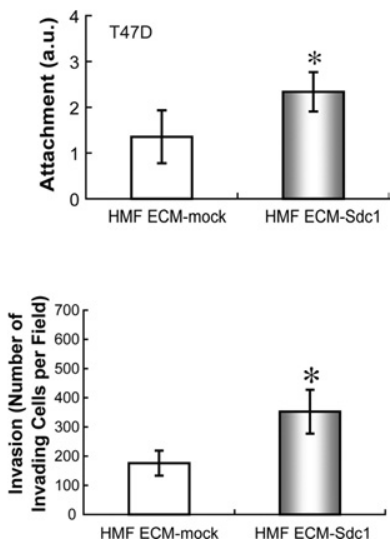

Figure 4. The ECMs produced by Sdc1-expressing fibroblasts promote the attachment and invasion of breast carcinoma cells. A: Attachment of MDAMB-231 and T47D cells to culture dishes precoated with 3D NIH-3T3 or HMF-derived ECM-mock and ECM-Sdc1 after 10 -minute incubation at $37^{\circ} \mathrm{C}$. The results were normalized by defining the average attachment to the FN-coated control vessel as 1 arbitrary unit. B: Invasion of MDA-MB-231 cells through ECM-mock and ECM-Sdc1 deposited in the inserts of basement membrane matrix (Matrigel) invasion chambers. Invasion is reported as the number of invading cells per field, as described in Materials and Methods ( $P$ $<0.01$ ). Significant differences at ${ }^{*} P<0.05$.

Sdc1 than ECM-mock from either fibroblast type (Figure 2D, $P<0.0001$ for both $\mathrm{NIH}-3 \mathrm{~T} 3$ and $\mathrm{HMF}$ ). These results show that Sdc1 in stromal fibroblasts plays a significant role in shaping ECM fiber architecture.

\section{ECM-Sdc1 Does Not Stimulate Breast Carcinoma Cell Proliferation}

The matrix microenvironment is a key determinant of carcinoma cell survival, proliferation, adhesion, migration, and invasion. ${ }^{30,31}$ We first examined the effects of ECMmock and ECM-Sdc1 on breast carcinoma cell proliferation. Two human breast carcinoma cell lines (the poorly differentiated highly invasive MDA-MB-231 cells and the better differentiated less invasive T47D cells) were plated onto the cell-free ECM-mock and ECM-Sdc1 from $\mathrm{NIH}$ $3 \mathrm{~T} 3$ and HMF and their growth was monitored throughout a 6 -day culture period. Our group previously determined in a co-culture model that Sdc1 expression in stromal fibroblasts stimulates carcinoma cell growth. ${ }^{17}$ However, culture of either carcinoma cell line in the cell-free ECM-Sdc1 did not accelerate growth rate (Figure 3), demonstrating that the mitogenic effect of Sdc1-positive fibroblasts was not retained after removal of the cells and Sdc1.

\section{ECM-Sdc1 Promotes Breast Carcinoma Cell Attachment and Invasion}

Although no growth difference was evident between carcinoma cells grown in ECM-mock versus ECM-Sdc1, the

ECM type did affect carcinoma cell attachment. Both MDA-MB-231 and T47D cells attached more readily to $\mathrm{NIH}-3 \mathrm{~T} 3$ or HMF ECM-Sdc1 than ECM-mock after 10 minutes of culture at $37^{\circ} \mathrm{C}$ (Figure $4 \mathrm{~A}$ ). To evaluate a potential role of ECMs in breast carcinoma invasion, Sdc1-positive and Sdc1-negative NIH 3T3 and HMF cells were cultured in the inserts of Matrigel invasion chambers for 7 days to allow for the deposition of their ECMs. Breast carcinoma cells were then plated into the cell-free ECMmock and ECM-Sdc1 and allowed to invade for 24 hours. Compared with the corresponding ECM-mock, significantly more MDA-MB-231 cells invaded through NIH 3 T3 or HMF ECM-Sdc1 (Figure 4B), indicating that the ECMs generated by the Sdc1-expressing fibroblasts have invasion-promoting properties. Consistent with prior reports, ${ }^{32}$ T47D cells did not invade through the chamber membranes (data not shown) and were not further considered in subsequent experiments.

\section{ECM-Sdc1 Promotes the Directional Migration of Breast Carcinoma Cells}

To determine the effect of ECMs on breast carcinoma cell migration, we performed time-lapse migration assays, observing MDA-MB-231 cell migration in real time for 5 to 6 hours. In HMF ECM-mock, most carcinoma cells moved with frequent, seemingly random changes in direction or oscillated in their original location (Figure 5A, left). In contrast, MDA-MB-231 cell movement in HMF ECM-Sdc1 was directionally persistent (Figure 5A, right). The migration patterns of MDA-MB-231 in the NIH-3T3-derived ECMs were similar to those observed in HMF-derived

A
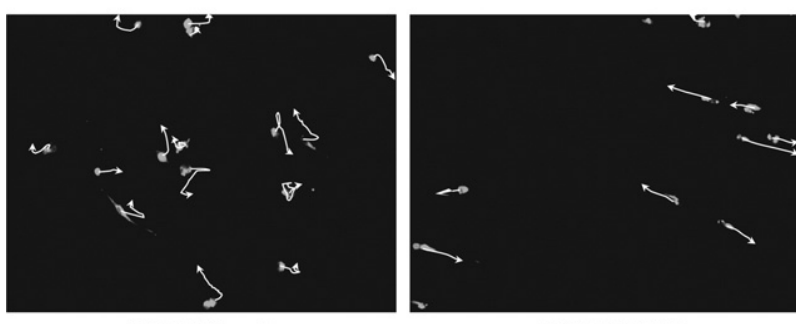

HMF ECM-Sdc1

B
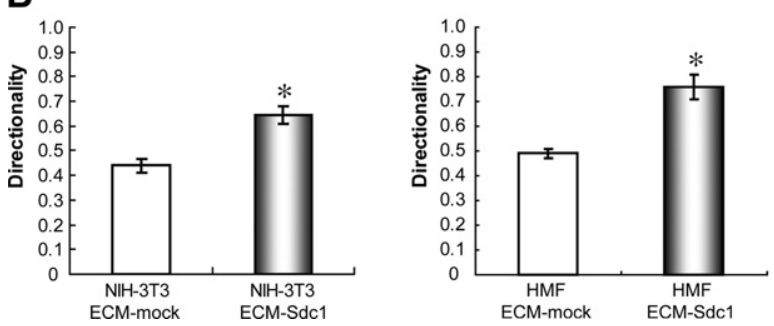

Figure 5. The ECM produced by Sdc1-expressing fibroblasts promotes directional migration of breast carcinoma cells. The migration behavior of MDA-MB-231 cells was assessed by time-lapse microscopy for 5 to 6 hours, and images were captured every 30 minutes. A: Typical tracks of MDA-MB-231 cells within HMF-derived ECM-mock and ECM-Sdc1 (original magnification, $\times 100$ ). B: The directionality of cell migration was determined as the ratio of the migration distance (net distance in a direct line from start to end point) to the total distance traveled. Significant differences at ${ }^{*} P<0.001$ 
ECMs (data not shown). Consistent with this visual impression, the directionality (ratio of net distance between starting point and end point to the total distance traveled) increased significantly in $\mathrm{NIH}-3 \mathrm{~T} 3$ or HMF ECM-Sdc1 versus ECM-mock (Figure 5B). Collectively, these data demonstrate that Sdc1-expressing fibroblasts assemble an ECM that supports the attachment, invasion, and directional migration of breast carcinoma cells.

\section{Increased FN Content in ECM-Sdc1 Promotes Breast Carcinoma Cell Attachment}

The ECM is a complex, organized meshwork of macromolecules composed of structural proteins (eg, fibrillar collagens), adhesive noncollagenous glycoproteins (eg, FN and laminin), and proteoglycans. We explored the possibility that the aberrant expression of Sdc1 in fibroblasts causes quantitative alterations in ECM composition in addition to the already demonstrated architectural changes. To analyze the ECM protein constituents, cellfree matrices were solubilized and subjected to Western blot analysis. The fibroblast-derived ECMs contained high levels of collagen I and FN, low levels of laminin I, and almost no vitronectin (data not shown). Among the analyzed proteins, only the FN level was elevated in ECM-Sdc1 from both NIH-3T3 and HMF compared with ECM-mock (Figure 6A).

To evaluate the contribution of increased FN deposition to the ECM-Sdc1 effect on carcinoma cells, we added increasing amounts of human $\mathrm{FN}$ to cell-free HMF ECM-mock and measured cancer cell attachment, invasion, and migration. Soluble FN bound to preexisting matrices, and $20 \mu \mathrm{g}$ of $\mathrm{FN}$ produced an FN content in ECM-mock equivalent to that found in ECM-Sdc1 (Figure $6 \mathrm{~B}$, left). To account for the possibility that FN added to cell-free ECMs may not be properly incorporated into the matrices, we also added FN to 3D fibroblast cultures during the ECM production stage. Other investigators ${ }^{11,33}$ showed that mammalian cells are capable of incorporating exogenously supplied FN into their ECMs. The addition of FN in this manner indeed increased the ECM FN content (Figure 6B, right) without changing the fiber architecture (data not shown). The increase of FN in ECM-mock augmented carcinoma cell attachment significantly, regardless of how exogenous $\mathrm{FN}$ was added (Figure 6C). This result is expected, considering that FN is an adhesion molecule that interacts with integrin and proteoglycan cell surface receptors. In contrast, the addition of FN had no effect on carcinoma cell invasion (Figure 6D) or migration (Figure 6E).

\section{ECM Fiber Architecture Engineering by Microcontact Printing Independent of Sdc1}

Because the increased FN content in ECM-Sdc1 did not explain the stimulation of carcinoma cell motility and directional movement observed in this matrix type, we explored the possibility that the parallel fiber architecture was causally involved. Microcontact printing was used to uncouple the fiber architecture from Sdc1 expression. A series of polydimethylsiloxane (PDMS) stamps with parallel line-patterned surface reliefs (a combination of different line widths and spacings ranging from 25 to 250 $\mu \mathrm{m})$ were "inked" with FN solutions, and the patterns were stamped onto culture dishes through conformal contact. Parallel FN lines with identical line widths and spacing of $150 \mu \mathrm{m}$ (Figure 7A, left) were best suited to ensure fibroblast attachment and force their parallel arrangement. The FN squares with a similar total surface area and the same spacing as the line pattern were also printed as controls (Figure 7A, right).

After a 20-minute incubation period, HMF cells attached selectively to FN patterns generated by microcontact printing (Figure 7B). The cells spread and grew along the FN patterns initially and then occupied the spaces between the patterns during the 7-day culture period (data not shown). Immunofluorescence staining of FN and collagen I demonstrated that HMF mock cells cultured on FN line-patterned surfaces generated ECMs (termed HMF ECM-mock/lines) with a parallel fiber architecture, mimicking the architecture seen in HMF ECMSdc1 (Figure 7C and data not shown). The fiber-to-fiber angles were significantly more acute $(P<0.001)$ in HMF

A

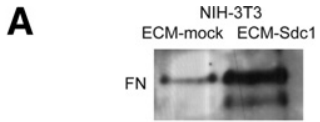

HMF

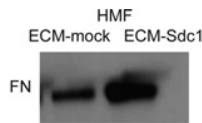

B

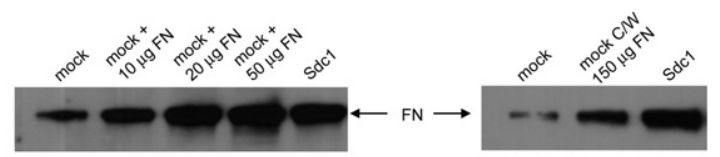

C
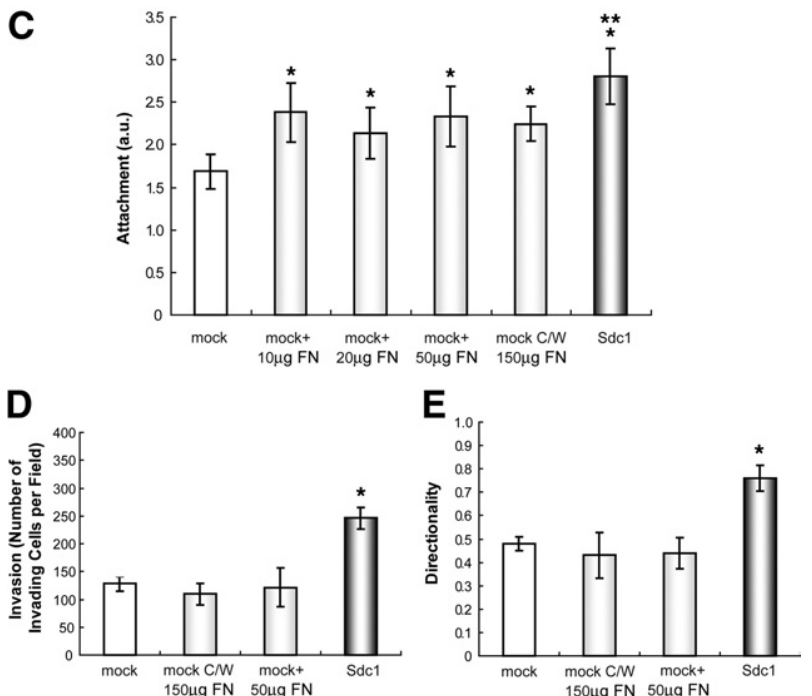

Figure 6. Increased FN content in HMF-derived ECM-Sdc1 promotes attachment of breast carcinoma cells but not invasion or directional movement. A Western blot analysis of FN in solubilized ECM-mock and ECM-Sdc1 derived from NIH-3T3 and HMF cells. B: Western blot analysis of FN in HMF ECMs. Left: The indicated amounts of soluble FN were added to cell-free ECMmock. Right: A total of $150 \mu \mathrm{g}$ FN was added to HMF mock cells during matrix production $(\mathrm{C} / \mathrm{W}$ indicates cultured with). C: Attachment of MDA-MB231 cells to HMF ECMs after a 10 -minute incubation period. ${ }^{*} P<0.05$ compared to mock; ${ }^{* *} P<0.05$ compared to mock $+20 \mu \mathrm{g}$ FN. D: Invasion of MDA-MB-231 cells through HMF ECMs. ${ }^{*} P<0.05$ compared to mock. E: Quantitative assessment of migration directionality of MDA-MB-231 cells within indicated HMF ECMs using time-lapse microscopy ${ }^{*} P<0.05 \mathrm{com}-$ pared to mock. 

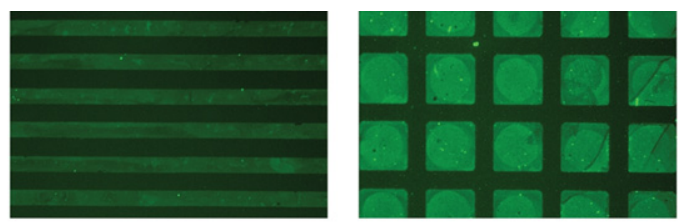

B
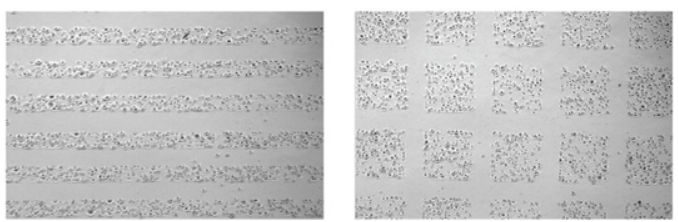
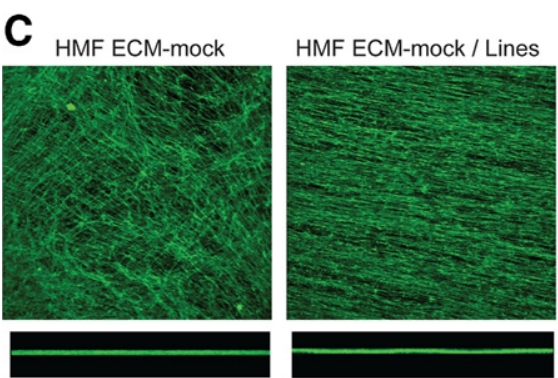

D

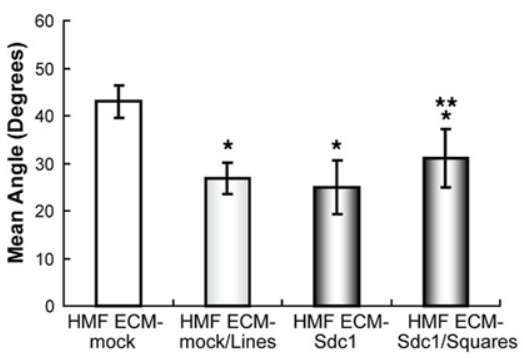

HMF ECM-Sdc1

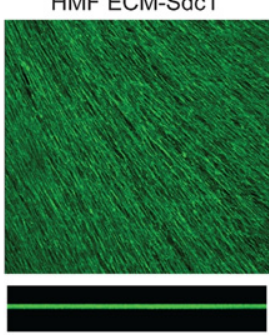

HMF ECM-Sdc1 / Squares

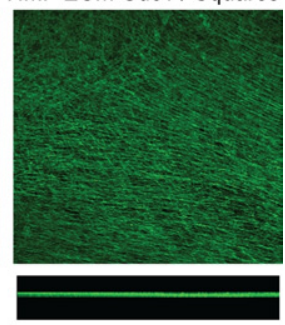

E

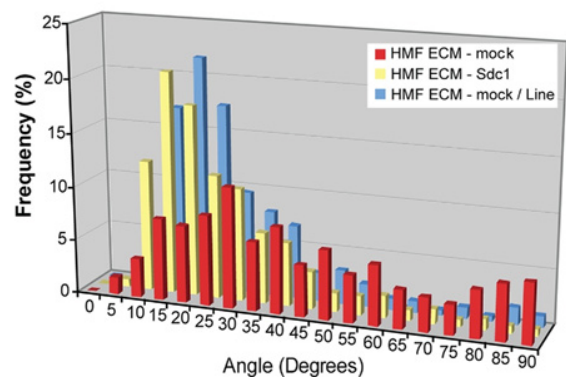

Figure 7. Generation of a parallel fiber architecture independent of Sdc1 by microcontact printing. A: Immunofluorescence images of $\mathrm{FN}$ line and square patterns produced on tissue culture surfaces using polydimethylsiloxane stamps (original magnification, $\times 40$ ). B: Phase-contrast images of HMF cells preferentially attached to the printed FN patterns (original magnification, $\times 40)$. C: Representative confocal images of immunofluorescently labeled FN fibers of ECMs from HMF mock and Sdc1 cells grown on patterned or regular tissue culture surfaces (original magnification, $\times 200$ ). Bottom: Z-axis views $\left(90^{\circ}\right.$ side views). D: Mean fiber-to-fiber angles of HMF ECMs. ${ }^{*} P<0.001$ compared to HMF ECMmock; ${ }^{* *} P<0.001$ compared to HMF ECM-Sdc1 E: Frequency distribution of fiber-to-fiber angles of indicated HMF ECMs.
ECM-mock/lines than in HMF ECM-mock and indistinguishable from HMF ECM-Sdc1 (Figure 7, D and E). The Sdc1-transfected HMFs on FN square-patterned surfaces (HMF ECM-Sdc1/squares) produced an ECM with wider angles than the same cells on unpatterned surfaces but with more acute angles than in ECM-mock (Figure 7, C and D).

\section{ECM Fiber Architecture Determines the Directionality of Breast Carcinoma Cell Migration}

In cell-free HMF ECMs with a parallel fiber arrangement, MDA-MB-231 cells migrated with increased directionality compared with HMF ECMs with random fiber organization, regardless of whether the parallel fibers were generated by Sdc1 expression or forced by microcontact printing (Figure 8A). Directionally persistent cell movement occurred primarily along, but also across, the parallel fibers and contrasted with the haphazard cell movement in the ECMs with randomly oriented fibers. In HMF ECM-Sdc1/squares, MDA-MB-231 cells moved in either random or directional patterns, likely dependent on the local ECM architecture. In contrast, the ECM fiber architecture had no significant effect on breast carcinoma cell attachment (Figure 8B).

\section{Discussion}

Sdc1 is aberrantly expressed by stromal fibroblasts in most infiltrating breast carcinomas. ${ }^{17,18}$ We have previously shown that Sdc1 expression in stromal fibroblasts stimulates breast carcinoma growth and angiogenesis. ${ }^{17,19,20}$ The present study demonstrates that stromal Sdc1 alters ECM composition and architecture in vivo and in vitro and that the altered ECM fiber architecture promotes the directional migration of breast carcinoma cells.

The ECM constitutes a complex macromolecular network of glycoproteins and proteoglycans that is essential for cell survival, proliferation, migration, and differentiation. During carcinoma cell invasion, the ECM undergoes extensive changes due to the abnormal synthesis of ECM components and their proteolytic remodeling. ${ }^{4,34}$ The accumulation of excess FN has been observed in the stroma of a variety of solid human tumors, ${ }^{34,35}$ and a significant correlation was observed between FN content and tumor stage/adverse outcome. Our results suggest that FN production in stromal fibroblasts is regulated by Sdc1. In our model, FN stimulates the attachment of breast carcinoma cells to the ECM but does not stimulate their migration behavior. This observation in 3D matrices contrasts with the biphasic relationship between migra- 

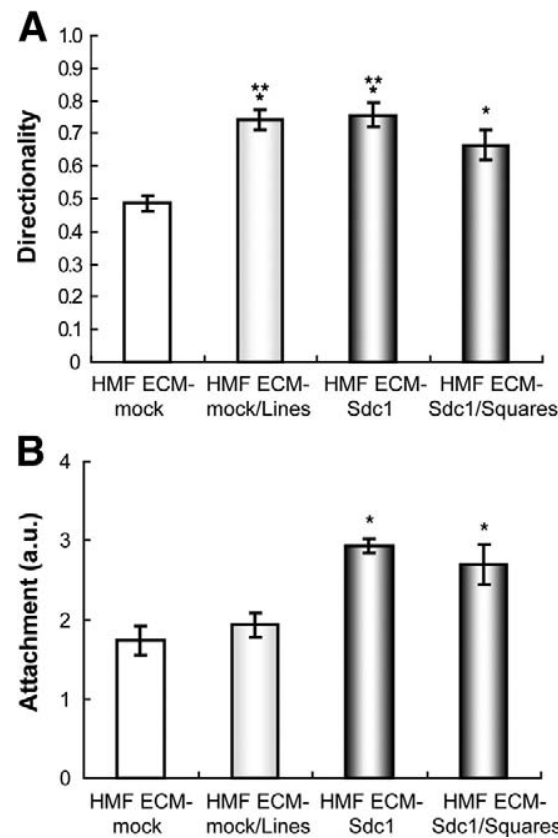

Figure 8. The ECM topography modulates MDA-MB-231 breast carcinoma cell migration. A: Directionality of carcinoma cell migration is dependent on matrix type (Materials and Methods provides the experimental details; ${ }^{*} \mathrm{P}<$ 0.05 compared to HMF-ECM-mock; ${ }^{* * *} P<0.05$ compared to HMF-ECM-Sdc1/ Squares. B: Attachment of carcinoma cells is dependent on matrix type (Materials and Methods provides the details). ${ }^{*} P<0.05$ compared to mock and mock/lines.

tion velocity and adhesion molecule (eg, FN and laminin) concentration observed under traditional two-dimentional conditions and may reflect the importance of the topographic presentation of adhesion ligands to the cell. ${ }^{36-38}$

In contrast to the intersecting meshwork of the ECM produced by Sdc1-negative fibroblasts, FN and collagen I fibers in ECM-Sdc1 are organized in parallel patterns. A parallel fiber arrangement was observed previously as a characteristic of ECM produced by primary carcinomaassociated fibroblasts of the skin. ${ }^{28}$ However, the consequence of this fiber arrangement for tumor cell behavior was unknown. Our study identifies ECM architecture as a determinant of directional cell migration. The parallel fiber architecture produced by Sdc1-positive fibroblasts is reminiscent of the collagen fiber signature identified in the transgenic Wnt-1 mouse mammary tumor model by Provenzano and colleagues. ${ }^{6}$ This group identified parallel collagen fibers perpendicular to the advancing edge of the tumors, which were spatially associated with carcinoma cells invading singly or collectively. Because of the inherent limitations of the model system, it was not clear whether the invasion was a consequence or cause of the fiber arrangement.

The molecular mechanism by which stromal Sdc1 modulates ECM assembly remains to be elucidated. An intact FN matrix is essential for the formation and stability of a mature collagen-containing ECM. ${ }^{39,40}$ Therefore, the regulation of $\mathrm{FN}$ fibril assembly may hold the key to understanding matrix organization. Fibronectin fibrillogenesis is a complex cell-mediated process that involves FN binding to cell surface receptors, FN-FN self-association, and interaction with the actin cytoskeleton. ${ }^{41,42}$ Fibronec- tin is secreted as compactly folded, disulfide-bonded dimers consisting of three types of repeating modules (types I, II and III). These FN dimers are initially inactive until they interact with integrins and other receptors at the cell surface. This binding interaction triggers intracellular signaling pathways, promotes actin cytoskeleton rearrangements, and eventually leads to conformational changes in FN that convert the molecule into an extended and active form. Stromal Sdc1 may modulate the program of FN fibrillogenesis during FN fibril initiation and elongation. The Sdc1 regulates the activities of several integrins, including $\alpha \vee \beta 3, \alpha \vee \beta 5$, and $\beta 4$ integrins. ${ }^{43-46}$ Although the FN matrix assembly appears to be initiated mainly by Arg-Gly-Asp-binding integrin $\alpha 5 \beta 1,47,48$ alternative FN-binding integrin receptors (eg, $\alpha \vee \beta 3, \alpha 4 \beta 1$, and $\alpha \vee \beta 1$ ) can also support this process once properly activated. ${ }^{33,49-52}$ It is possible that Sdc1 induction activates integrins other than $\alpha 5 \beta 1$ and initiates an alternative assembly pathway, thus resulting in an ECM that is structurally/compositionally different from that assembled under the control of $\alpha 5 \beta 1$. It is also possible that stromal Sdc1 directly participates in the FN matrix assembly. The compact conformation of $\mathrm{FN}$ dimers is maintained by intramolecular interactions involving the type $\mathrm{III}_{12-14}$ repeats of $\mathrm{FN}{ }^{53,54}$ The type $\mathrm{II}_{12-14}$ repeats, also known as heparin II binding domain, interact with the HS chains from various members of the Sdc family. ${ }^{55}$ Binding of Sdc1 with heparin II may facilitate unfolding of dimeric FNs and expose FN self-assembly (FN-binding) sites, thus promoting $\mathrm{FN}$ deposition and fibrillogenesis.

Evidence supporting the importance of the biophysical attributes of the ECM for breast carcinoma cell behavior is compelling. Dense rigid physical properties suppress tubulogenesis and possibly stimulate invasion of welldifferentiated breast carcinoma cells in collagen gels by stimulating the activity of the small GTPase Rho. ${ }^{56,57}$ The cell-derived matrices used in our study are more pliable than the basement membrane or collagen gels commonly used to assess 3D matrix effects on breast carcinoma cell behavior. ${ }^{58}$ Therefore, we favor a model in which the fiber topography rather than matrix rigidity governs cell invasion in fibroblast-derived matrices. A dissection of the molecular mechanism of cell migration regulation by parallel ECM fibers was beyond the scope of this study but may involve Rac1, a member of the Rho family of GTPases. Inhibition of Rac1 can switch the migration mode of fibroblasts and epithelial cells from random to more directionally persistent. ${ }^{59}$ The induction of cell migration is required for tumor invasion and metastasis $^{60-62}$ and, therefore, it is plausible that ECMs with parallel fiber organization facilitate tumor cell spread. In summary, this study demonstrates a novel pathway of how aberrant expression of Sdc1 in stromal fibroblasts might enhance tumor progression.

\section{Acknowledgments}

We thank other members of the Friedl Laboratory, especially Gui Su, for help and suggestions; Dianhua Qiao for amplification of the mouse Sdc1-containing pcDNA3 
plasmid; Dr Caroline Alexander for providing paraffin blocks of Wnt1-induced mouse mammary tumors; Jessica Kueck and Carolyn Pehlke for participating in the ECM angle measurements; Kathleen Schell, supervisor of the UWCCC Flow Cytometry Facility; Dr Peter Kaufmann for helpful discussions; and Dr Korise Rasmusson for help with manuscript preparation.

The contents of this article do not represent the views of the Department of Veterans Affairs or the United States government.

\section{References}

1. Bauer G: Elimination of transformed cells by normal cells: a nove concept for the control of carcinogenesis. Histol Histopathol 1996, 11:237-255

2. Kuperwasser C, Chavarria T, Wu M, Magrane G, Gray JW, Carey L, Richardson A, Weinberg RA: Reconstruction of functionally normal and malignant human breast tissues in mice. Proc Natl Acad Sci U S A 2004, 101:4966-4971

3. Barsky SH, Green WR, Grotendorst GR, Liotta LA: Desmoplastic breast carcinoma as a source of human myofibroblasts. Am J Pathol 1984, 115:329-333

4. Schor SL, Ellis IR, Jones SJ, Baillie R, Seneviratne K, Clausen J, Motegi K, Vojtesek B, Kankova K, Furrie E, Sales MJ, Schor AM, Kay RA: Migration-stimulating factor: a genetically truncated onco-fetal fibronectin isoform expressed by carcinoma and tumor-associated stromal cells. Cancer Res 2003, 63:8827-8836

5. Tuxhorn JA, Ayala GE, Smith MJ, Smith VC, Dang TD, Rowley DR Reactive stroma in human prostate cancer: induction of myofibroblast phenotype and extracellular matrix remodeling. Clin Cancer Res 2002, 8:2912-2923

6. Provenzano PP, Eliceiri KW, Campbell JM, Inman DR, White JG, Keely $\mathrm{PJ}$ : Collagen reorganization at the tumor-stromal interface facilitates local invasion. BMC Med 2006, 4:38

7. Provenzano PP, Inman DR, Eliceiri KW, Knittel JG, Yan L, Rueden CT, White JG, Keely PJ: Collagen density promotes mammary tumor initiation and progression. BMC Med 2008, 6:11

8. Lopes CC, Dietrich CP, Nader HB: Specific structural features of syndecans and heparan sulfate chains are needed for cell signaling Braz J Med Biol Res 2006, 39:157-167

9. Tkachenko E, Rhodes JM, Simons M: Syndecans: new kids on the signaling block. Circ Res 2005, 96:488-500

10. Zimmermann $P$, David G: The syndecans, tuners of transmembrane signaling. FASEB J 1999, 13 Suppl:S9-S100

11. Klass CM, Couchman JR, Woods A: Control of extracellular matrix assembly by syndecan-2 proteoglycan. J Cell Sci 2000, 113(Pt 3) 493-506

12. Saoncella S, Echtermeyer F, Denhez F, Nowlen JK, Mosher DF, Robinson SD, Hynes RO, Goetinck PF: Syndecan-4 signals cooperatively with integrins in a Rho-dependent manner in the assembly of focal adhesions and actin stress fibers. Proc Natl Acad Sci U S A 1999, 96:2805-2810

13. Wilcox-Adelman SA, Denhez F, Goetinck PF: Syndecan-4 modulates focal adhesion kinase phosphorylation. J Biol Chem 2002, 277 : 32970-32977

14. Ilic D, Kovacic B, Johkura K, Schlaepfer DD, Tomasevic N, Han Q, Kim JB, Howerton K, Baumbusch C, Ogiwara N, Streblow DN, Nelson JA, Dazin P, Shino Y, Sasaki K, Damsky CH: FAK promotes organization of fibronectin matrix and fibrillar adhesions. J Cell Sci 2004 117:177-187

15. Wierzbicka-Patynowski I, Schwarzbauer JE: Regulatory role for SRC and phosphatidylinositol 3-kinase in initiation of fibronectin matrix assembly. J Biol Chem 2002, 277:19703-19708

16. Sanderson RD, Hinkes MT, Bernfield M: Syndecan-1, a cell-surface proteoglycan, changes in size and abundance when keratinocytes stratify. J Invest Dermatol 1992, 99:390-396

17. Maeda T, Alexander CM, Friedl A: Induction of syndecan-1 expression in stromal fibroblasts promotes proliferation of human breast cancer cells. Cancer Res 2004, 64:612-621
18. Stanley MJ, Stanley MW, Sanderson RD, Zera R: Syndecan-1 expres sion is induced in the stroma of infiltrating breast carcinoma. Am J Clin Pathol 1999, 112:377-383

19. Su G, Blaine SA, Qiao D, Friedl A: Shedding of syndecan-1 by stromal fibroblasts stimulates human breast cancer cell proliferation via FGF2 activation. J Biol Chem 2007, 282:14906-14915

20. Maeda T, Desouky J, Friedl A: Syndecan-1 expression by stromal fibroblasts promotes breast carcinoma growth in vivo and stimulates tumor angiogenesis. Oncogene 2006, 25:1408-1412

21. Harvey JM, Clark GM, Osborne CK, Allred DC: Estrogen receptor status by immunohistochemistry is superior to the ligand-binding assay for predicting response to adjuvant endocrine therapy in breast cancer. J Clin Oncol 1999, 17:1474-1481

22. Cukierman E: Cell migration analyses within fibroblast-derived 3-D matrices. Methods Mol Biol 2005, 294:79-93

23. Cukierman E: Preparation of Extracellular Matrices Produced by Cultured Fibroblasts. Edited by JS Bonifacino, M Dasso, J LippincottSchwartz, JB Harford, KM Yamada. New York, John K. Wiley \& Sons 2002, pp 10.19.11-10.19.14

24. Bernard A, Delamarche E, Schmid H, Michel B, Bosshard HR, Biebuyck $\mathrm{H}$ : Printing patterns of proteins. Langmuir 1998, 14:2225-2229

25. Baba F, Swartz K, van Buren R, Eickhoff J, Zhang Y, Wolberg W, Friedl A: Syndecan-1 and syndecan-4 are overexpressed in an estrogen receptor-negative, highly proliferative breast carcinoma subtype. Breast Cancer Res Treat 2006, 98:91-98

26. Bauer M, Eickhoff JC, Gould MN, Mundhenke C, Maass N, Friedl A Neutrophil gelatinase-associated lipocalin (NGAL) is a predictor of poor prognosis in human primary breast cancer. Breast Cancer Res Treat 2008, 108:389-397

27. Junqueira LC, Bignolas G, Brentani RR: Picrosirius staining plus polarization microscopy, a specific method for collagen detection in tissue sections. Histochem J 1979, 11:447-455

28. Amatangelo MD, Bassi DE, Klein-Szanto AJ, Cukierman E: Stromaderived three-dimensional matrices are necessary and sufficient to promote desmoplastic differentiation of normal fibroblasts. Am J Pathol 2005, 167:475-488

29. Cukierman E: Preparation of extracellular matrices produced by cultured fibroblasts. Edited by JB Harford and KM Yamada. Philadelphia, Lippincott-Schwartz, 2002, pp 10.19.11-10.19.14

30. Weigelt B, Bissell MJ: Unraveling the microenvironmental influences on the normal mammary gland and breast cancer. Semin Cancer Biol 2008, 18:311-321

31. Nelson CM, Bissell MJ: Of extracellular matrix, scaffolds, and signaling: tissue architecture regulates development, homeostasis, and cancer. Annu Rev Cell Dev Biol 2006, 22:287-309

32. Thompson EW, Paik S, Brunner N, Sommers CL, Zugmaier G, Clarke R, Shima TB, Torri J, Donahue S, Lippman ME, Martin GR, Dickson RB: Association of increased basement membrane invasiveness with absence of estrogen receptor and expression of vimentin in human breast cancer cell lines. J Cell Physiol 1992, 150:534-544

33. Wu C, Keivens VM, O'Toole TE, McDonald JA, Ginsberg MH: Integrin activation and cytoskeletal interaction are essential for the assembly of a fibronectin matrix. Cell 1995, 83:715-724

34. Wilhelm O, Hafter R, Coppenrath E, Pflanz MA, Schmitt M, Babic R, Linke R, Gossner W, Graeff H: Fibrin-fibronectin compounds in human ovarian tumor ascites and their possible relation to the tumor stroma. Cancer Res 1988, 48:3507-3514

35. Moro L, Colombi M, Molinari Tosatti MP, Barlati S: Study of fibronectin and mRNA in human laryngeal and ectocervical carcinomas by in situ hybridization and image analysis. Int J Cancer 1992, 51:692-697

36. DiMilla PA, Stone JA, Quinn JA, Albelda SM, Lauffenburger DA Maximal migration of human smooth muscle cells on fibronectin and type IV collagen occurs at an intermediate attachment strength. J Cell Biol 1993, 122:729-737

37. Goodman SL, Risse G, von der Mark K: The E8 subfragment of laminin promotes locomotion of myoblasts over extracellular matrix. J Cell Biol 1989, 109:799-809

38. Palecek SP, Loftus JC, Ginsberg MH, Lauffenburger DA, Horwitz AF Integrin-ligand binding properties govern cell migration speed through cell-substratum adhesiveness. Nature 1997, 385:537-540

39. Sottile J, Hocking DC: Fibronectin polymerization regulates the composition and stability of extracellular matrix fibrils and cell-matrix adhesions. Mol Biol Cell 2002, 13:3546-3559 
40. Velling T, Risteli J, Wennerberg K, Mosher DF, Johansson S: Polymerization of type I and III collagens is dependent on fibronectin and enhanced by integrins alpha 11 beta 1 and alpha 2beta 1. J Biol Chem 2002, 277:37377-37381

41. Wierzbicka-Patynowski I, Schwarzbauer JE: The ins and outs of fibronectin matrix assembly. J Cell Sci 2003, 116:3269-3276

42. Mao Y, Schwarzbauer JE: Fibronectin fibrillogenesis, a cell-mediated matrix assembly process. Matrix Biol 2005, 24:389-399

43. Beauvais DM, Burbach BJ, Rapraeger AC: The syndecan-1 ectodomain regulates alphavbeta3 integrin activity in human mammary carcinoma cells. J Cell Biol 2004, 167:171-181

44. McQuade KJ, Beauvais DM, Burbach BJ, Rapraeger AC: Syndecan-1 regulates alphavbeta5 integrin activity in B82L fibroblasts. J Cell Sci 2006, 119:2445-2456

45. Ogawa T, Tsubota Y, Hashimoto J, Kariya Y, Miyazaki K: The short arm of laminin gamma2 chain of laminin-5 (laminin-332) binds syndecan-1 and regulates cellular adhesion and migration by suppressing phosphorylation of integrin beta4 chain. Mol Biol Cell 2007, 18:1621-1633

46. Beauvais DM, Ell BJ, McWhorter AR, Rapraeger AC: Syndecan-1 regulates alphavbeta3 and alphavbeta5 integrin activation during angiogenesis and is blocked by synstatin, a novel peptide inhibitor. J Exp Med 2009, 206:691-705

47. Fogerty FJ, Akiyama SK, Yamada KM, Mosher DF: Inhibition of binding of fibronectin to matrix assembly sites by anti-integrin (alpha 5 beta 1) antibodies. J Cell Biol 1990, 111:699-708

48. Wu C, Bauer JS, Juliano RL, McDonald JA: The alpha 5 beta 1 integrin fibronectin receptor, but not the alpha 5 cytoplasmic domain, functions in an early and essential step in fibronectin matrix assembly. J Biol Chem 1993, 268:21883-21888

49. Yang JT, Hynes RO: Fibronectin receptor functions in embryonic cells deficient in alpha 5 beta 1 integrin can be replaced by alpha $V$ integrins. Mol Biol Cell 1996, 7:1737-1748

50. Wu C, Hughes PE, Ginsberg MH, McDonald JA: Identification of a new biological function for the integrin alpha $v$ beta 3: initiation of fibronectin matrix assembly. Cell Adhes Commun 1996, 4:149-158

51. Wennerberg K, Lohikangas L, Gullberg D, Pfaff M, Johansson S, Fassler R: Beta 1 integrin-dependent and -independent polymerization of fibronectin. J Cell Biol 1996, 132:227-238
52. Sechler JL, Cumiskey AM, Gazzola DM, Schwarzbauer JE: A nove RGD-independent fibronectin assembly pathway initiated by alpha4beta1 integrin binding to the alternatively spliced $V$ region. J Cell Sci 2000, 113( Pt 8):1491-1498

53. Johnson KJ, Sage H, Briscoe G, Erickson HP: The compact conformation of fibronectin is determined by intramolecular ionic interactions. J Biol Chem 1999, 274:15473-15479

54. Hynes RO: The dynamic dialogue between cells and matrices: implications of fibronectin's elasticity. Proc Natl Acad Sci U S A 1999, 96:2588-2590

55. Tumova S, Woods A, Couchman JR: Heparan sulfate chains from glypican and syndecans bind the Hep II domain of fibronectin similarly despite minor structural differences. J Biol Chem 2000, 275:9410-9417

56. Wozniak MA, Desai R, Solski PA, Der CJ, Keely PJ: ROCK-generated contractility regulates breast epithelial cell differentiation in response to the physical properties of a three-dimensional collagen matrix J Cell Biol 2003, 163:583-595

57. Paszek MJ, Zahir N, Johnson KR, Lakins JN, Rozenberg GI, Gefen A Reinhart-King CA, Margulies SS, Dembo M, Boettiger D, Hammer DA Weaver VM: Tensional homeostasis and the malignant phenotype. Cancer Cell 2005, 8:241-254

58. Green JA, Yamada KM: Three-dimensional microenvironments modulate fibroblast signaling responses. Adv Drug Deliv Rev 2007, 59 1293-1298

59. Pankov R, Endo Y, Even-Ram S, Araki M, Clark K, Cukierman E, Matsumoto K, Yamada KM: A Rac switch regulates random versus directionally persistent cell migration. J Cell Biol 2005, 170:793-802

60. Vicente-Manzanares M, Webb DJ, Horwitz AR: Cell migration at a glance. J Cell Sci 2005, 118:4917-4919

61. Ridley AJ, Schwartz MA, Burridge K, Firtel RA, Ginsberg MH, Borisy G, Parsons JT, Horwitz AR: Cell migration: integrating signals from front to back. Science 2003, 302:1704-1709

62. Zijlstra A, Lewis J, Degryse B, Stuhlmann H, Quigley JP: The inhibition of tumor cell intravasation and subsequent metastasis via regulation of in vivo tumor cell motility by the tetraspanin CD151. Cancer Cell 2008, 13:221-234 